\title{
HORIZONTES DE LA EDICIÓN ELECTRÓNICA CON FINES DIDÁCTICOS
}

\author{
Myriam Ferreira* \\ María García Sánchez \\ Marta Ortiz ${ }^{* \star x}$
}

\begin{abstract}
RESUMEN: La edición digital está cambiando la forma de acercarse a los libros en todos los ámbitos del conocimiento, incluida la educación. Por eso, este artículo propone un acercamiento teórico a las perspectivas de la edición electrónica con fines didácticos. El texto se divide en tres secciones. En un primer momento trataremos de realizar un acercamiento al momento actual en que se encuentran las ediciones académicas y cómo este tipo de edición permite adaptaciones en el entorno escolar y con fines educativos. En la segunda parte se describirá el proceso seguido en la introducción de estas ediciones académicas en las aulas, desde los CD adjuntos a libros de texto hasta los nuevos libros digitales, multimodales e interactivos. Y finalmente se propone una reflexión en torno a el aprovechamiento de las TIC/TAC en la red y su equivalencia en los centros educativos para la práctica de la edición digital en el aula (EDEA).
\end{abstract}

PALABRAS CLAVE: Edición Electrónica. Didáctica. Humanidades Digitales. TIC/TAC. EDEA.

\section{La edición digital académica: perspectivas para la edición digital educativa}

Con la llegada de la edición en formato digital las entidades que participan del proceso editorial de un texto toman nuevos roles y deben atender a diversos imperativos. Como comentan Price y Siemens (2013, p. 8), "the digital turn has also changed and reinvigorated a foundational element of scholarly work: textual editing". En efecto, no solo cambian las figuras de autor y lector, sino que el editor académico, aquel que quiere ofrecer un texto lo más fiable posible, debe adaptarse a la nueva demanda del público y a las nuevas herramientas que el formato digital ofrece. Con los avances tecnológicos, la idea que tenemos de los textos se modifica a gran velocidad:

\footnotetext{
*Universidad Internacional de La Rioja, España. E-mail: Myriam.ferreira@unir.net

** Universidad Internacional de La Rioja, España. E-mail: maria.garsanchez@unir.net

*** Universidad Internacional de La Rioja, España. E-mail: marta.ortiz@unir.net
}

\section{(cc) EY-NC-ND}

Esta obra está licenciada com uma licença Creative Commons. 
El texto ya no es una entidad cerrada, sino un objeto abierto y penetrable, permeable a participaciones e influjos propios de una sociedad red como la que se está estructurando. El documento electrónico reemplaza las ideas de secuencialidad y causalidad por las de una actividad integrada y continua. En realidad, en lugar de hablar de productos, podríamos hablar de procesos en los que la obra se mantiene en una apertura permanente (CORDÓN, 2011, p. 42).

Así, siguiendo a Cordón (2011), encontramos cuatro niveles en los que se produce el impacto de la digitalización en el sector editorial: en los sistemas operativos; en la gestión y manipulación del contenido; en el marketing y la distribución; y en el suministro de contenidos. Pero no debemos confundir al editor como intermediario 'comercial' o distribuidor, aquel que posee una editorial, con el editor académico, quien fija un texto con un criterio determinado para ofrecerlo al público en una versión fiable que siga lo más fielmente posible la voluntad del autor. Nos interesa aquí la figura del editor académico, aquel que lleva a cabo tanto ediciones de textos para un público especializado y profesional, como ediciones de los mismos textos con fines educativos o divulgativos.

En ambos casos, el editor debe atender a la naturaleza de la obra en formato digital. Para ello, es importante distinguir entre las obras con contenido tradicional que se adaptan al formato electrónico, de las obras que se crean expresamente para ser leídas o asimiladas en formato digital. Tal y como apunta Romero (2013, p. 105), los nuevos soportes informáticos afectan a la literatura en tres aspectos: "la edición de textos literarios, la didáctica de las literaturas a través de internet y las nuevas aportaciones críticas basadas en las nuevas teorías del cibertexto". Este último aspecto se relaciona con la literatura digital, es decir, aquellas obras literarias que aprovechan el formato electrónico como un añadido, y no solo un medio, a su propia creación; aquellas obras que se crean para el medio digital.

Resulta interesante acercarse a las posibles vías de entrada a los textos literarios digitales por las enormes posibilidades que ofrece en términos didácticos. Según Romero (2013, p. 111-114), existen tres vías de entrada a este tipo de textos: la lectura hipertextual, realizada a través de enlaces, de una manera más o menos lineal; la lectura ecfrástica, que ofrece diversos enlaces al mismo tiempo, con lo que se rompe 
la lectura lineal, de manera que el hipertexto se convierte en hipermedia; y la lectura de la serendipia, que permite que el lector se deje llevar "por la gravitación de un mundo virtual bidimensional o tridimensional" (ROMERO, 2013, p. 114). Se trataría de una lectura generada por el mismo ordenador y que nos introduce en una especie de entropía digital.

Sobra decir que estas tres vías se le ofrecen no solo al autor de un texto literario digital, sino también al editor del mismo. Si bien un texto literario creado para ser leído o consumido en la web puede ofrecer de por sí alguna o todas estas vías de entrada, un texto tradicional que un editor decide pasar a formato digital también puede ser intervenido de la misma manera. Los enlaces hipertextuales, las decisiones múltiples que se le ofrecen al lector, los distintos niveles de lectura más o menos especializados..., son mecanismos de los que el editor dispone para realizar una edición especializada, si va dirigida a un público muy determinado, o divulgativa y didáctica, si lo que quiere es introducir estos elementos en el aula.

Pero, ¿a qué nos referimos cuando hablamos de edición académica? Quizá la definición que ofrece Price (2008, [s.p.]) nos ayude a comprender este concepto:

\footnotetext{
By scholarly edition, I mean the establishment of a text on explicitly stated principles and by someone with specialized knowledge about textual scholarship and the writer or writers involved. And edition is scholarly both because of the rigor with which the text is reproduced or altered and because of the expertise brought to bear on the task and in the offering of suitable introductions, notes, and textual apparatus.
}

El concepto de archivo ha cambiado también con la aparición de ediciones electrónicas: en un contexto digital, una edición es solo una parte o una pieza de un archivo, puesto que este puede incluir muchos más objetos digitales además de lo que es propiamente la edición de sus textos. Una de las mayores ventajas de la edición electrónica, en ese sentido, es la de la capacidad que permite: en una edición electrónica podemos incluir todo tipo de contenido multimedia que en las ediciones tradicionales no se incluían. Del mismo modo, esto hace que las responsabilidades del equipo editorial crezcan drásticamente, porque siempre va a haber una 
perspectiva desde la que se edita: por ejemplo un proyecto editorial centrado en un autor, o desde la perspectiva de género, etc. (PRICE, 2008).

La responsabilidad del grupo que edita será la de ofrecer una guía para el lector; desde esa perspectiva desde la que se edita, no se debe incluir todo lo que se pueda sin más, sino que debe haber un criterio y se debe ayudar al lector a abarcar todo el archivo que se le ofrece. De hecho, según Price (2008), la mejor edición electrónica es la que combina bien lo que tiene el archivo como base de datos y la narrativa que nosotros como editores podemos ofrecer para que ese archivo sea legible, accesible, atractivo y tenga un aparato crítico accesorio que complemente al texto. En esto radica la principal diferencia entre colecciones o bibliotecas digitales y ediciones académicas: la colección o biblioteca es acumulación y catalogación de objetos digitales; la edición académica ofrece algo más. Y en ese mismo sentido, una edición con fines didácticos ofrecerá también su propia narrativa, en un registro apropiado para el público al que va dirigido.

Una característica adicional de las ediciones electrónicas es su potencial para ser un trabajo siempre en desarrollo: un permanente work-in-progress. El lado negativo de esto es determinar cuándo podemos considerar que una edición electrónica es estable y puede asimilarse por un determinado público.

\section{La introducción del libro digital en el aula}

La introducción de los libros digitales en el aula fue temprana ya que, en ocasiones, fue promovida por las propias editoriales de libros de texto. En efecto, la aparición del libro electrónico ofreció la posibilidad de enriquecer los manuales con material complementario que se distribuía en un soporte digital (disquete, CD o DVD), como una herramienta para complementar y ampliar los contenidos de las asignaturas. A estos materiales complementarios hay que añadir otras publicaciones en formato digital con fines didácticos, como diccionarios o enciclopedias (por ejemplo, Encarta o la Enciclopedia Británica). Estos libros digitales eran muy sencillos y básicos, con escasa interactividad y con una preponderancia del texto escrito sobre otros soportes. 
Además, en su mayor parte estos contenidos eran visualizados en ordenadores según Cordón (2011), en 2010 el porcentaje de población que leía libros digitales en el ordenador era del $97.3 \%$ (p. 218)-, lo cual hacía que no favorecieran una lectura fácil ni cómoda. Como señalan Sagredo y Espinosa, en los archivos soportados en CD "apenas existían las características esenciales del libro tradicional, es decir, su ergonomía y accesibilidad universales" (FERNÁNDEZ; TEMIÑO, 2000, p. 9-10). Por esa razón, estos materiales se utilizaban como fuentes de consulta y no para una lectura continuada: Cordón citaba un estudio realizado en 2008 donde se concluía que la ratio de uso de estos libros eran unos 13 minutos por sesión y unas 8 páginas de visionado, lo cual demostraba que "su uso era considerado más como un complemento que como una solución autónoma” (CORDÓN, 2010, p. 51). Aun así, estos libros electrónicos se siguieron editando porque permitían distribuir un gran número de contenidos con un coste mucho más reducido que un libro tradicional, y más aún cuando, con la extensión de Internet, los CDs o DVDs fueron poco a poco sustituidos por archivos accesibles en Internet mediante suscripción, lo que suponía una forma de abaratar aún más los costes y garantizar la actualización de la información.

Esta situación cambió radicalmente con la generalización de los e-readers, muy perfeccionados técnicamente gracias a la aparición de la tinta electrónica, así como con la difusión de las Tablets, que ofrecían la posibilidad de una lectura de los libros digitales mucho más cómoda (CORDÓN, 2011). El libro digital experimentó en esos momentos un aumento espectacular, de modo que no solo se planteaba como un complemento al libro de papel, sino que empezó a plantearse como su sustituto.

La escuela no podía permanecer impermeable a este cambio. Muy pronto, en muchos países se promovieron campañas para introducir los libros electrónicos en las aulas (DÍAZ, 2013), debido a sus innegables ventajas. Los libros digitales se adecuaban al carácter de nativos digitales de los alumnos mejor que los libros de texto tradicionales, tenían un coste inferior, se consideraban un medio más económico y suponían una reducción drástica del peso de las mochilas escolares, un problema que puede afectar 
a la salud de los niños, como han alertado especialistas médicos (AGUILAR; SÁNCHEZ; BUENROSTRO, 2007).

Las editoriales de libros de texto se fueron viendo, por lo tanto, en la necesidad de adaptar sus propios libros de texto a los nuevos dispositivos electrónicos. Se empezaron a crear y difundir libros de texto electrónicos que pudieran utilizarse en PC, portátil, e-reader, Tablet o PDI. El crecimiento de este sector en los últimos años, que ha pasado de 598 títulos en 2008 a 3.667 en 2013, ha sido vertiginoso y superior a cualquier otro sector editorial. Sin embargo, a pesar de esta producción tan espectacular, los resultados de las ventas fueron mucho muy limitados: si la producción de libros creció en un $259.5 \%$, la venta solo creció un $21.7 \%$ (Observatorio de la Lectura y el Libro, 2014).

¿Por qué ha costado tanto la difusión de los libros electrónicos digitales? Se suele indicar que las causas principales serían la falta de herramientas adecuadas (no todos los alumnos disponen de un dispositivo electrónico) y la falta de preparación tecnológica del profesorado, así como el miedo a que estos materiales, aunque motivadores, no mejoren el rendimiento escolar de los estudiantes (MORAL-PÉREZ; VILLALUSTRE, 2014). Pero otro elemento que se debe tener en cuenta es la escasa funcionalidad del libro de texto electrónico concebido como simple digitalización del manual en papel. Como indicaba Adell (2011), "si en lugar de libros de texto de papel usamos libros de texto digitales, prácticamente idénticos, y los utilizamos de la misma manera que los de papel, es lógico que los resultados sean los mismos que antes" ( $p$. 13). Una valoración que también recogían, con más extensión, en su estudio Minelli de Oliveira, Camacho Martí y Gisbert Cervera (2014).

Por ello, como indicaban estas autoras, los libros de texto electrónicos tienen que "responder a los requisitos comunicativos y tecnológicos de la sociedad digitalizada" (OLIVEIRA; MARTÍ; CERVERA, 2014, p. 89). Unos requisitos que Vázquez definía en siete principios metodológicos: simplicidad, didáctica, dinamicidad, legibilidad, interactividad, hipertextualidad y flexibilidad (GUTIÉRREZ, 2011, p. 340). Para poder 
desarrollar estos principios, los libros de texto electrónicos han tenido que ir incluyendo las siguientes funcionalidades:

- Sustitución de un texto plano por un texto enriquecido: por medio de enlaces, se permite ampliar información sobre algún aspecto concreto y ponerlo en relación con otros temas similares. De este modo, la lectura deja de ser unívoca y permite una mayor o menor adquisición de información según los intereses del lector. Además, el libro digital facilita la consulta de palabras en un diccionario o el uso de un traductor a medida que se va leyendo, algo muy necesario en educación.

- Carácter multimodal de los contenidos: el libro digital permite la inserción de imágenes interactivas, vídeos, podcasts (archivos de audio), actividades interactivas o autocorregibles, etc.

- Mayor posibilidad de interacción: existe la posibilidad de escribir y subrayar los propios documentos, así como de interactuar con compañeros mediante correo electrónico, wifi o bluetooth, compartir archivos o documentos, enviar actividades a los profesores para su corrección...

- Posibilidad de integrar apps específicas: los alumnos disponen en un mismo dispositivo de herramientas como agenda escolar, calculadoras, lienzos para dibujar, mapas, representaciones de biología, etc.

- Educación más personalizada, por la posibilidad de ampliar o reforzar los contenidos. La flexibilidad de este modelo permite incluso mantener diferentes niveles de aprendizaje entre los alumnos (ARMAÑANZAS, 2010).

Es evidente que este tipo de libros digitales están también orientando la elección del dispositivo en el que se van a utilizar, anteponiendo la Tablet a otros dispositivos como los e-readers o los PCs, que no permiten la visualización de algunos elementos o no permiten la integración de apps.

Las editoriales han adaptado estas características en sus libros de texto de formato digital. Un repaso rápido por editoriales como Edelvives, Anaya, SM, Casals o Edebé 
nos permite ver cómo los contenidos interactivos y multimodales se han convertido en la pauta en la elaboración de contenidos para libros de texto ${ }^{1}$.

Una innovación de este calibre está despertando gran interés entre los especialistas, que sitúan el libro electrónico como uno de los cambios más interesantes que van a afectar a la educación en los próximos años. Es lógico que el prestigioso informe Horizon Report, orientado a la Educación Superior, en su edición de 2014 considere a las publicaciones electrónicas dentro de la "Master List" de tecnologías en observación. Pero este informe va más allá y considera que los libros electrónicos son uno de los factores que harán posible un importante cambio en la educación de los próximos años: el aula invertida o flipped classroom. En el aula invertida, la iniciativa en el aprendizaje la toman los alumnos, que acceden a un gran número de recursos en busca de información, mientras el profesor guía el aprendizaje y resuelve las dudas que van surgiendo (JOHNSON et al., 2014, p. 45-48).

En este modelo didáctico, los libros digitales juegan un importante papel. Y, como es lógico, los libros de texto digitales deben recoger esta posibilidad. Un ejemplo al respecto lo podemos ver ya en un proyecto de la editorial Edelvives, dirigido a la Educación Secundaria: \#somoslink, donde, junto a todas las potencialidades señaladas respecto a los libros de texto digitales, se incluyen propuestas de innovación educativa, entre las que se cita expresamente la flipped classroom ${ }^{2}$.

Sin embargo, este cambio en la concepción de los contenidos no es la única revolución que está afectando a las ediciones de libros electrónicos con fines didácticos. Cordón (2011) ya indicaba que el libro electrónico está facilitando la irrupción en el mercado editorial de nuevos autores que no precisan de grandes inversiones económicas para editar sus publicaciones. Y este fenómeno está llegando también al mundo de la educación. La facilidad y el abaratamiento de costes que suponen las ediciones digitales permiten que los profesores creen sus propios contenidos y los utilicen para

\footnotetext{
1 La información sobre estos proyectos se encuentra en http://www.edelvives.com/, http://www.anayaeducacion.es/proyecto-digital.php, http://www.smlir.com/, http://www.ecasals.net/ y http://www.edebe.com/libros-digitales/

${ }^{2}$ La información sobre este proyecto se encuentra en http://www.somoslink.com/
} 
el aula. Existen incluso proyectos para compartir, bajo licencias Creative Commons, estos contenidos, en proyectos como Marea Verde ${ }^{3}$.

Flipped classroom, contenidos multimodales, mayor interacción, educación personalizada, mayor autonomía para el profesor a la hora de escoger los contenidos... Todos estos conceptos, que en gran medida están siendo facilitados por los libros electrónicos, nos permiten intuir que lo que el libro digital está aportando a la didáctica no es solo un nuevo soporte, sino un cambio global en cómo entendemos la educación.

\section{Las TIC /TAC y la edición digital en el aula (EDEA)}

Cuando hablamos de la edición digital en el aula, en adelante (EDEA), hablamos de la enseñanza - aprendizaje de la edición digital como estrategia para el desarrollo e interacción de las facultades racionales y creativas de los estudiantes, vinculadas a los procesos de alfabetización digital en el aula. En este sentido se hace necesaria una reflexión sobre el proceso de enseñanza-aprendizaje de la EDEA, ya que éste, no solo está relacionado con las tecnologías de la información y la comunicación (TIC), también lo está con las tecnologías del aprendizaje y el conocimiento (TAC).

Las TIC han cambiado el mundo de la educación a través de la creación de nuevas tecnologías que posibilitan el acceso global a múltiples contenidos y formas de comunicarse, han modificado los modelos tradicionales de enseñanza y comunicación, constantemente nos plantean nuevos retos en el proceso de alfabetización digital impulsando nuevos modelos de enseñanza en el ámbito educativo. Las TIC han modificado y alterado los roles del profesor y del alumno en el aula. Ahora el profesor es un coordinador, un entrenador, un guía, de la enseñanzaaprendizaje centrada en el sujeto que aprende, y construye su propia experiencia, desarrollando nuevas habilidades y destrezas para relacionarse con diversos entornos, a través de distintos medios.

\footnotetext{
${ }^{3}$ La información sobre este proyecto se encuentra en http://librosmareaverde.tk/ 
Más allá de esta realidad, nuevas investigaciones recogen como es el caso de Experiencias educativas en las aulas del siglo XXI - Innovación con TIC, más de un centenar de experiencias docentes, que demuestra que las TIC, no introducen ninguna innovación didáctica por sí mismas, y menos sí las seguimos aplicando en el aula basándonos en metodologías tradicionales. "Si en lugar de libros de texto de papel usamos libros de texto digitales, prácticamente idénticos, y los utilizamos de la misma manera que los de papel, es lógico que los resultados sean los mismos que antes" (ADELL, 2011, p. 13). Así mismo y en esta misma dirección, comenta Ávila Muñoz (1999), que "deben ofertarse cursos que faciliten al profesorado el aprendizaje de utilización de las TIC en las aulas (...), que fomente el trabajo colaborativo entre alumnado y profesorado, que aporte ideas sobre materiales en la red (...); y (...) que identifiquen y difundan las buenas prácticas".

En este escenario y como respuesta a este -sin sentido- surgen las TAC ${ }^{4}$ ofreciendo al profesorado un marco de acción y comprensión tanto del uso pedagógico y reflexivo de las TIC, como de sí mismos, y dando luz al profesorado como agentes autónomos con cualidades individuales y esenciales del conocimiento, capaces de influir significativamente en el adecuado (o inadecuado) proceso de integración de las TIC en el aula. Las TAC proponen un tratamiento, personal, abierto y colaborativo que intervienen en el proceso de producción de experiencias multimodales, que llenan de sentido la forma que tienen los usuarios de percibir y comprender los aspectos esenciales de las TIC. Las TAC según Lozano (2011) "van más allá de aprender a usar las TIC y apuestan por explorar estas herramientas tecnológicas y ponerlas al servicio del aprendizaje y de la adquisición de conocimiento". No obstante, tal y como reflejan las investigadoras Espuny-Vidala, Coiduras, Gisbert-Cervera, GonzálezMartínez:

La formación en/con TAC requiere una actualización constante, y, por tanto, a menudo las preguntas recurrentes son estas: ¿me lo ofrece la Administración? ¿Yo estoy preparado? ¿Me compensa de alguna manera: promoción/dedicación, horaria/compensación, económica/oportunidades de formación/prestigio...? Evidentemente, la respuesta negativa a estas respuestas implica desazón y abandono. (2010, p.15).

\footnotetext{
${ }^{4}$ http://www.tpack.org/

Texto Digital, Florianópolis, Santa Catarina, Brasil, v. 12, n. 1, p. 4-19, jan./jun. 2016. ISSNe: 1807-9288.
} 
Esta reflexión sobre las TIC/TAC, a propósito de la EDEA, nos llevan a valorar los procesos retroalimentarios como diferenciadores en el proceso de enseñanzaaprendizaje de las TIC/TAC -a la vez que igualarlos- en su nivel de importancia en el aula, ya que la tendencia general es la de dar por sentados estos procesos implícitos en las TIC/TAC, y más aún sobreponiendo la importancia de las unas sobre las otras. En la investigación Explorando la percepción de estudiantes y profesores sobre el libro de texto electrónico en Educación Primaria, se observó que:

\begin{abstract}
Los estudiantes no siempre son capaces de distinguir el libro de texto electrónico de otros recursos digitales: el ordenador, el Ipad o el lector de libros de texto electrónicos parecían conformar lo que los estudiantes identificaban como el objeto de aprendizaje, no las fuentes semióticas que los educadores ponen a su disposición en los distintos elementos de tecnología que utilizan. (OLIVEIRA; MARTí; CERVERA, 2014, p. 93)
\end{abstract}

Como hemos podido ver, no solo se trata interactuar con las TIC sino de ir más allá en busca del conocimiento esencial que permita a los profesores intervenir positivamente el los procesos de práctica de la EDEA y a su vez la EDEA contribuya al desarrollo integral de las facultades racionales y creativas de los alumnos, aunque todavía "la percepción de los usuarios de los libros de texto electrónicos depende de la cultura institucional en la que están inmersos" (p. 94). Y todavía, al libro electrónico se le está dando el mismo uso que al libro tradicional, y ciertamente, sus posibilidades de éxito como una nueva alternativa en el aula, está en estrecha relación con la trascendencia de las prácticas tradicionales de lectura y la creciente oferta de recursos en la red. Y más cierto aún que todavía que "existen desafíos reales que las editoriales deben superar para que el libro de texto electrónico no se convierta en una moda pasajera" (p. 94).

A pesar de los pros y de los contras en el inminente proceso de cambio educativo al que estamos asistiendo, y mientras tanto, podemos en función de la práctica de la EDEA, aprovecharnos de los recursos humanos que los nuevos lectores han desarrollado: 
(...) nuevas destrezas que les permiten adaptarse a la sobrecarga y encontrarse cómodos ante semejante bombardeo de bits: las noticias, los diccionarios y las compras en línea, el e-mail, las redes sociales, los blogs y los microblogs, las descargas de vídeo, música, juegos y un largo etc. se combinan de manera fluida en nuestras breves incursiones en el ciberespacio. (CARCEDO; JORGE, 2013, p.142).

También, podemos aprovecharnos de los recursos tecnológicos, que la red ha desarrollado, como son los diversos programas inteligentes, divertidos y especializados para la autoedición y autopublicación textual e hipertextual de libros, que cuentan con una serie de herramientas compatibles con tabletas, teléfonos inteligentes u otros dispositivos, que suelen ser muy útiles para el trabajo en el aula y más allá del aula, que ponen a la disposición de alumnos y profesores un soporte de ayuda que les permite resolver dudas y problemillas que surgen sobre la marcha en la práctica de la EDEA.

Por otra parte tambien podemos aprovechar y poner en marcha algunas buenas prácticas para el desarrollo de la EDEA, tales como:

- Concebir la organización de un plan diario y materiales curriculares una clave como para la interacción alumnos -profesor en el proceso de la EDEA.

- lluminar los procedimientos de autonomía, reflexión y conocimiento de la EDEA.

- Sopesar el uso equivalente de las TIC/TAC en la práctica de la EDEA.

- Adecuar las dinámicas a la experiencia espacio-temporal de la EDEA.

- Facilitar la aportación de ideas y herramientas al proceso de la EDEA.

- Evitar los posibles riesgos propios de la práctica de EDEA.

- Valorar el equilibrio de las funciones cognitivas y emocionales durante la práctica de la EDEA.

- Favorecer la integración de diferentes procedimientos metodológicos y

- Adoptar un enfoque retroalimentario entre los distintos tipos de evaluación en el proceso de la EDEA.

- Generar motivos y estrategias con el fin de la continuidad de la de la EDEA. 
La enseñanza-aprendizaje de la EDEA, como venimos viendo, no radica solo en la cantidad y/o calidad de los dispositivos TIC, que los centros educativos y otros entornos educativos ponen a disposición de profesores y alumnos, también reside en la equivalencia e incorporación de las TAC a los procesos de práctica de la EDEA. Y en cuanto a la práctica de la EDEA solo resaltar su gran potencial humano, ya que visto lo visto, todo producto de la imaginación, es editable y publicable, a través de la red. De ahí la importancia de los procesos retroalimentarios de las TIC/TAC y su aprovechamiento como herramienta posibilitadora de la práctica de la EDEA que profesores y alumnos, del mundo entero, día tras día, firman con sus vidas en el aula.

\section{AN OUTLOOK ON ELECTRONIC EDITING FOR EDUCATIONAL PURPOSES}

ABSTRACT: Digital publishing is changing the way we draw near to books in all areas of knowledge, including education. Because of that, this article suggests a theoretical approach to perspectives of electronic publishing for educational purposes.

The text is divided into three sections. Firstly, we will try to carry out an approach to the current situation of academic editions and how this type of publishing allows adaptations in the school environment and with educational purposes. Secondly, the process followed to introduce these academic editions in classroom will be described; from the CDs attached to the textbooks to the new digital books, multimodal and interactive. And finally, a reflection is proposed about the use of the ICT/TPACK on the web and its equivalence to the practice of digital edition and publishing in classroom (DEPIC), at educational centres.

KEYWORDS: Electronic Edition. Didactics. Digital Humanities. ICT /TPACK. DEPIC.

\section{Referencias}

ADELL, Jordi. Pedagogía 2.0. En: ORTEGA, José. et al. (Coord.). Experiencias educativas en las aulas del siglo XXI: Innovación con TIC. Madrid: Fundación Telefónica, 2011. p. 11-14. Disponible en:

<http://www.apuntesdelengua.com/ExperienciasXXI.pdf>. Acceso en: 16 abr. 2015.

AGUILAR, José Manuel Ruano; SÁNCHEZ, Ruth Palafox; BUENROSTRO, Noé García. Las mochilas en los escolares y su asociación con dolor de espalda. Acta Médica Grupo Ángeles, Ciudad de México, v. 5, n. 4, p. 225-227, 2007. Disponible en: <http://www.medigraphic.com/pdfs/actmed/am-2007/am074h.pdf>. Acceso en: 16 abr. 2015. 
ARMAÑANZAS, Emy. El libro electrónico, una gran herramienta para la cultura y la educación. En: PÉREZ TORNERO, José Manuel. Actas del Congreso

Eurolberoamericano. Alfabetización mediática y culturas digitales. Sevilla:

Universidad de Sevilla, 2010. Disponible en:

$<$ http://www.gabinetecomunicacionyeducacion.com/files/adjuntos/El\%20libro\%20elec tr\%C3\%B3nico,\%20una\%20gran \%20herramienta\%20para\%20la\%20educaci\%C3\% B3n.pdf>. Acceso en: 16 abr. 2015.

ESPUNY-VIDALA, Cinta; GISBERT -CERVERA, Mercé; GONZÁLEZ-MARTÍNEZ, Juan.; COIDURAS, Jordi. Los seminarios TAC. Un reto de formación para asegurar la dinamización de las TAC en las escuelas. Edutec-e. Revista electrónica de tecnología educativa, $\mathrm{n}^{\circ}$ 34. Universitat Rovira i Virgili (Tarragona-España), Universitat de Lleida(Lerida-España) 2010. p.15. Disponible en: $<$ http://edutec.rediris.es/Revelec2/revelec34/seminarios_tac_formacion_dinamizacion _escuelas.html> Acceso en 22 abr.2015

CARCEDO, Pilar García; JORGE, María Goicoechea de. Presentación de modelos de experimentación y resultados. Alicia a través de la pantalla: estudio de la lectura literaria en el siglo XXI. Alicia a través de la pantalla: lecturas literarias en el siglo XXI. Alicante : Biblioteca Virtual Miguel de Cervantes. 2013. p. 142. Disponible en: $<$ http://www.cervantesvirtual.com/obra/alicia-a-traves-de-la-pantalla-estudio-de-lalectura-literaria-en-el-siglo-xxi-presentacion-de-modelos-de-experimentacion-yresultados/>. Acceso en: 21 abr. 2015.

CORDÓN GARCÍA, José Antonio. De la lectura ensimismada a la lectura colaborativa: nuevas topologías de la lectura en el entorno digital. En: GÓMEZ DÍAZ, Raquel; AGUSTÍN LACRUZ, María del Carmen (coord.). Polisemias visuales: Aproximaciones a la alfabetización visual en la sociedad intercultural. Salamanca: Universidad de Salamanca, 2010. p. 39-84. Disponible en: <http://eprints.rclis.org/15441/1/lecturaensimismada.pdf>. Acceso en: 16 abr. 2015.

CORDÓN GARCÍA, José Antonio; GÓMEZ dÍAZ, Raquel; ALONSO ARÉVALO, Julio. Guttenberg 2.0: La revolución de los libros electrónicos. Gijón: Trea, 2011.

DÍAZ, Adriana Plasencia. Los libros de texto electrónicos gratuitos: un acercamiento a la realidad en México. Revista Internacional de Estudios sobre Sistemas Educativos, Ciudad de México, v. 1, n. 1-2, p. 69-86, 2013. Disponible en: <http://www.riesed.org/revista/index.php/RIESED/article/view/7/23>. Acceso en: 16 abr. 2015.

FERNÁNDEZ, Félix Sagredo; TEMIÑO, María Blanca Espinosa. Del libro, al libro electrónico-digital. Cuadernos de documentación multimedia, Madrid, v. 9, n. 3, 2000. Disponible en:

<http://pendientedemigracion.ucm.es/info/multidoc/multidoc/revista/num9/cine/sagred o.htm>. Acceso en: 16 abr. 2015.

GUTIÉRREZ, María Azucena Vázquez. Contenidos digitales en el aula. En: ORTEGA, José Hernández. et al. (Coord.). Experiencias educativas en las aulas del 
siglo XXI: Innovación con TIC. Madrid: Fundación Telefónica, 2011. p. 339-343.

Disponible en: <http://www.apuntesdelengua.com/ExperienciasXXI.pdf>. Acceso en: 16 abr. 2015.

JOHNSON, Larry; BECKER, Samantha Adams; ESTRADA, Victoria; FREEMAN, Alex. NMC Horizon Report: Edición Educación Superior 2014. Madrid: Universidad Internacional de La Rioja y Cátedra UNESCO en E-Learning, 2014.

LOZANO, Roser. Las TIC/TAC: de las tecnologías de la información y comunicación a las tecnologías del aprendizaje y del conocimiento. Instituto Latinoamericano de la comunicación educativa, México. DF. 2011. Disponible en:

$<$ http://www.thinkepi.net/las-tic-tac-de-las-tecnologias-de-la-informacion-ycomunicacion-a-las-tecnologias-del-aprendizaje-y-del-conocimiento>. Acceso en: 21 abr. 2015.

MORAL-PÉREZ, María Esther Del; VILLALUSTRE, Lourdes. Libros digitales: valoraciones del profesorado sobre el modelo de formación bimodal. Revista Latinoamericana de Tecnología Educativa, Cáceres, v. 13, n. 1, p. 89-99, 2014. Disponible en: <http://relatec.unex.es/article/view/1291/867>. Acceso en: 16 abr. 2015.

MUÑOZ, Patricia Avila. Aprendizaje con nuevas tecnologías. Paradigma emergente. Instituto Latinoamericano de la comunicación educativa, México. DF. 1999. p. 7. Disponible en:

<http://investigacion.ilce.edu.mx/panel_control/doc/c37aprendizaje.pdf>. Acceso en: 21 abr. 2015.

OBSERVATORIO DE LA LECTURA Y EL LIBRO. El sector del libro en España. 2012-2014. Madrid: Ministerio de Educación, Cultura y Deporte, 2014. Disponible en: <http://www.mcu.es/libro/docs/MC/Observatorio/pdf/Sectorlibro_abril2014.pdf>. Acceso en: 16 abr. 2015.

OLIVEIRA, Janaina Minelli de; MARTÍ, María del Mar Camacho; CERVERA, Mercè Gisbert. Explorando la percepción de estudiantes y profesor sobre el libro de texto electrónico en Educación Primaria. Comunicar: Revista científica iberoamericana de comunicación y educación, Huelva, n. 42, p. 87-95, 2014. Disponible en:

<http://dialnet.unirioja.es/descarga/articulo/4525705/1.pdf>. Acceso en: 16 abr. 2015.

PRICE, Kenneth. Electronic Scholarly Editions. En: SIEMENS, Ray; SCHREIBMAN, Susan (eds.). A Companion to Digital Literary Studies. Oxford: Blackwell, 2008. Disponible en: <http://www.digitalhumanities.org/companionDLS/>. Acceso en: 20 abr. 2015.

PRICE, Kenneth M.; SIEMENS, Ray. Literary Studies in the Digital Age: An Envolving Anthology. New York: MLA, 2013. Disponible en:

<https://dlsanthology.commons.mla.org/>. Acceso en: 20 abr. 2015. 
ROMERO LÓPEZ, Dolores. Vías de entrada a la lectura de literatura digital con fines didácticos. En: GOICOECHEA, María (ed.). Alicia a través de la pantalla: lecturas literarias en el siglo XXI. Madrid: Fundación Germán Sánchez Ruipérez, 2013.

Recebido em: 19/05/2016. Aceito em: 26/06/2016. 